\title{
Analysis of EHMT1 expression and its correlations with clinical significance in esophageal squamous cell cancer
}

\author{
XIAOJIAO GUAN ${ }^{1 *}$, XINWEN ZHONG ${ }^{2 *}$, WANFU MEN ${ }^{2}$, SHULEI GONG ${ }^{2}$, LIN ZHANG $^{2}$ and YUCHEN HAN ${ }^{1,3}$ \\ ${ }^{1}$ Department of Pathology, Basic Science College, China Medical University; Departments of ${ }^{2}$ Thoracic Surgery and \\ ${ }^{3}$ Pathology, The First Affiliated Hospital of China Medical University, Shenyang, Liaoning, P.R. China
}

Received June 11, 2013; Accepted August 28, 2013

DOI: $10.3892 / \mathrm{mco} .2013 .207$

\begin{abstract}
Esophageal squamous cell carcinoma (ESCC) is a highly aggressive malignancy, requiring effective biomarkers for prognosis and therapeutic responsiveness. Histone H3K9 methyltransferases (EHMT1 and EHMT2) are global genome organizers, which are crucial for maintaining the balance state of cells in a tissue-specific manner. It was previously suggested that EHMT1 expression is a predictor of prognosis in several malignant tumors; however, the prognostic significance of EHMT1 expression in ESCC has not been determined. A cohort of 50 ESCC cases and 46 paired normal esophageal tissue samples were evaluated to assess the levels of EHMT1 expression by immunohistochemistry and reverse transcription-polymerase chain reaction. The SPSS software package was used for statistical data analysis. A significantly upregulated EHMT1 expression was observed in squamous preinvasive lesions and ESCC compared to the matched normal esophageal epithelia (52.0 vs. $21.7 \%$, respectively). The expression of EHMT1 was correlated with tumor grade $(\mathrm{G})$, depth of invasion (T) and lymph node metastasis (N) in ESCC. EHMT1 overexpression was found to be associated with poor cancer-specific survival in squamous cell carcinomas $\left(\chi^{2}=3.922, \mathrm{P}=0.048\right)$. The expression of EHMT1 was identified as an independent prognostic factor for overall survival in ESCC patients. In conclusion, EHMT1 expression is upregulated in ESCC and early preinvasive esophageal squamous lesions and the overexpression of EHMT1 is associated with poor prognosis in ESCC. Therefore, the expression of EHMT1 may be an effective prognostic biomarker for ESCC.
\end{abstract}

Correspondence to: Professor Yuchen Han, Department of Pathology, The First Affiliated Hospital of China Medical University, 155 Nanjingbei Street, Shenyang, Liaoning 110001, P.R. China

E-mail: xwzhong@mail.cmu.edu.cn

${ }^{*}$ Contributed equally

Key words: histone methyltransferase, EHMT1, esophageal squamous cell cancer, prognosis

\section{Introduction}

Esophageal squamous cell carcinoma (ESCC) is a highly aggressive malignancy, with early lymphatic and hematogenous metastasis. The treatment of ESCC primarily relies on classical treatment modalities including surgery, radiotherapy and chemotherapy, or a combination of these methods; however, the outcome has not improved significantly (1). The poor prognosis of ESCC may improve with a greater understanding of the molecular basis of this disease, with a focus on molecular risk markers that may lead to improved detection and treatment strategies.

ESCC develops through the accumulation of genetic and epigenetic abnormalities, evolving from preinvasive lesions to invasive esophageal cancer, with the malignant cells commonly exhibiting abnormal histone expression. EHMT1 is an important subunit of H3K9 methyltransferases. The highly similar euchromatic H3K9 methyltransferases EHMT1 and EHMT2 (also referred to as GLP and G9a, respectively) form a heteromeric complex and the loss of either one significantly reduces mono- and dimethylation of $\mathrm{H} 3 \mathrm{~K} 9$, a marker of silent euchromatin (2), which is a procedure crucial for the transcription, signal transduction, proliferation and differentiation of cells $(3,4)$. EHMT1 has also been recognized for its ability to methylate histone H1.4, as well as other non-histone proteins, including itself (5-7). EHMT1 binds to its H3K9mel products via ankyrin repeat domains, which contain a hydrophobic cage present in methyllysine-binding modules of diverse folds. Therefore, it is hypothesized that the disruption of EHMT1 may also result in major alterations in gene expression patterns that may contribute to cell canceration.

It was previously suggested that disordered histone methylation may induce tumorigenesis (8). However, our knowledge of the expression and clinical significance of EHMT1 in ESCC and peripheral tissues is limited; therefore, thorough investigation of the EHMT1 expression in ESCC and the normal peripheral esophageal epithelium is required. In our study, we analyzed EHMT1 expression in a cohort of 50 patients and demonstrated that EHMT1 expression was significantly upregulated in squamous preinvasive lesions and ESCC, with the expression of EHMT1 in ESCC being associated with tumor grade, depth of invasion and lymph node metastasis. This clinical case study verified that the expression of EHMT1 predicted poor cancer-specific survival in human ESCC. This study also demonstrated that the expression of EHMT1 may be 
Table I. Association between EHMT1 expression and clinicopathological data of esophageal squamous cell cancer.

\begin{tabular}{|c|c|c|c|c|c|c|}
\hline \multirow{2}{*}{$\begin{array}{l}\text { Clinicopathological } \\
\text { parameters }\end{array}$} & \multirow[b]{2}{*}{ No. } & \multicolumn{3}{|c|}{ EHMT1 expression } & \multirow[b]{2}{*}{$\chi^{2}$} & \multirow[b]{2}{*}{ P-value } \\
\hline & & Positive & Negative & Positivity rate (\%) & & \\
\hline Age (years) & & & & & 0.002 & 0.963 \\
\hline$\leq 60$ & 21 & 11 & 10 & 52.4 & & \\
\hline$>60$ & 29 & 15 & 14 & 51.7 & & \\
\hline Gender & & & & & 0.020 & 0.887 \\
\hline Male & 40 & 21 & 19 & 52.5 & & \\
\hline Female & 10 & 5 & 5 & 50.0 & & \\
\hline Tumor grade & & & & & 12.426 & $0.002^{\mathrm{a}}$ \\
\hline G1 & 14 & 6 & 8 & 42.9 & & \\
\hline $\mathrm{G} 2$ & 20 & 6 & 14 & 30.0 & & \\
\hline G3-4 & 16 & 14 & 2 & 87.5 & & \\
\hline Depth of invasion & & & & & 9.992 & $0.002^{\mathrm{a}}$ \\
\hline $\mathrm{T} 1-2$ & 18 & 4 & 14 & 22.2 & & \\
\hline T3-4 & 32 & 22 & 10 & 68.8 & & \\
\hline Lymph node metastasis & & & & & 14.753 & $0.001^{\mathrm{a}}$ \\
\hline No & 28 & 8 & 20 & 28.6 & & \\
\hline $\mathrm{N} 1$ & 16 & 14 & 2 & 87.5 & & \\
\hline N2-3 & 6 & 4 & 2 & 66.7 & & \\
\hline Tumor stage & & & & & 32.902 & $0.000^{\mathrm{a}}$ \\
\hline I & 10 & 2 & 8 & 20.0 & & \\
\hline II & 18 & 6 & 12 & 33.3 & & \\
\hline III-IV & 22 & 18 & 4 & 81.8 & & \\
\hline
\end{tabular}

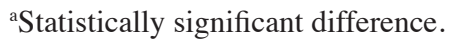

a novel prognostic biomarker for ESCC and a potential target for clinical treatment.

\section{Materials and methods}

Clinical samples. A total of 50 patients who underwent resection for ESCC between 2003 and 2007 at the Department of Thoracic Surgery, the First Affiliated Hospital of China Medical University, were included in this study. None of these patients had received chemotherapy or radiotherapy prior to surgery. The tumor specimens were either cut immediately after removal from the resected esophagus, frozen in liquid nitrogen and stored at $-80^{\circ} \mathrm{C}$, or fixed in $10 \%$ formalin and embedded in paraffin for histopathological analysis. All the cases were independently classified by two experienced pathologists as ESCC, according to the guidelines of the World Health Organization. The criteria of the tumor-node-metastasis (TNM) staging system were used to classify the clinicopathological factors and clinical stages of esophageal cancer (defined by the International Union Against Cancer TNM Classification of Malignant Tumors, 7th edition, 2009). The patients provided signed informed consent and were subjected to close follow-up. The patient sample comprised 40 men and 10 women, with a mean age of 63 years (range, 41-84 years) at the time of surgery. A summary of the clinicopathological characteristics is presented in Table I. The median follow-up time after surgery was 30 months (range, 3-60 months).
This study was approved by the Human Research Ethics Committee of China Medical University, which is accredited by the National Council on Ethics in Human Research.

Immunohistochemistry. Immunohistochemical studies on EHMT1 were performed on formalin-fixed, paraffin-embedded tissue sections obtained from the 50 ESCC patients. The tissue sections were deparaffinized and boiled in $0.01 \mathrm{~mol} / 1$ sodium citrate buffer $(\mathrm{pH}=6.0)$ in a 1,000-Watt microwave oven for $10 \mathrm{~min}$ to retrieve cell antigens. Rabbit monoclonal anti-EHMT1 was used as primary antibody (1:100 dilution; Abcam, Cambridge, UK). The tissue sections were immunohistochemically stained using the avidin-biotin-peroxidase method and counterstained with hematoxylin.

Evaluation of immunostaining for EHMT1. The staining was scored by two independent investigators who were blinded to the patient outcomes. The sections were evaluated at low magnification (x100) to identify areas of even EHMT1 staining. The percentage of positively stained cells was calculated among >400 tumor cells. The expression of EHMT1 was scored by staining intensity and percentage of cells exhibiting nuclear staining as follows: negative expression, negative or weak staining in all the tumor cells or moderate staining in $<25 \%$ of the tumor cells; positive expression, moderate or strong staining in $>25 \%$ of the tumor cells. Any discrepancies 
Table II. EHMT1 expression in esophageal squamous cell cancer and normal esophageal squamous epithelium.

\begin{tabular}{lcccccr}
\hline \multirow{2}{*}{ Group } & & \multicolumn{4}{c}{ EHMT1 expression } & \\
\cline { 3 - 6 } & No. & Positive & Negative & Positivity rate (\%) & $\chi^{2}$ & P-value \\
\hline Esophageal cancer & 50 & 26 & 24 & 52.0 & 9.361 & $0.002^{\text {a }}$ \\
Normal esophageal epithelium & 46 & 10 & 36 & 21.7 & & \\
\hline
\end{tabular}

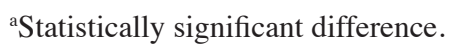
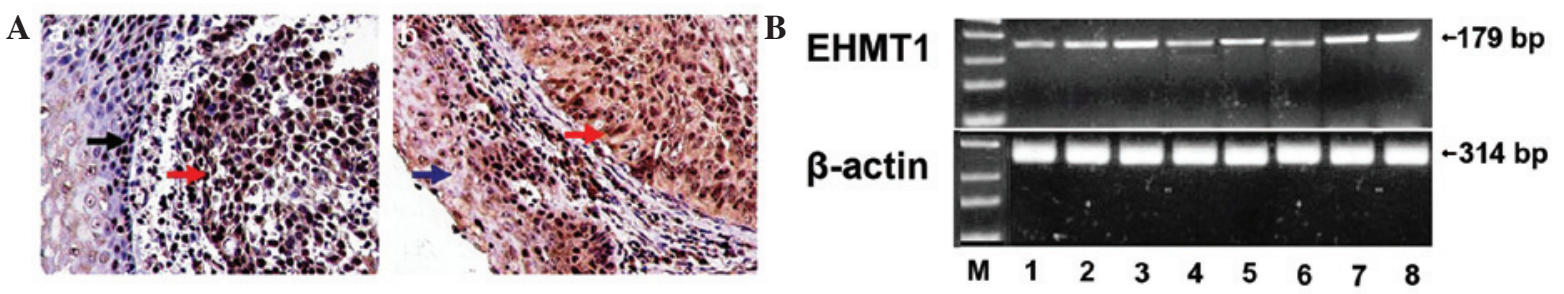

Figure 1. EHMT1 expression in esophageal squamous cell carcinoma (ESCC). (A) Immunohistochemical results: (A-a) EHMT1 expression in normal esophageal epithelium (black arrow) and ESCC (red arrow); (A-b) EHMT1 expression in atypical hyperplastic esophageal epithelium (blue arrow) and ESCC (red arrow); bar, $20 \mu \mathrm{m}$. (B) RT-PCR analysis of EHMT1 in ESCC. mRNA expression levels in: lane M, marker; lane 1, normal esophageal tissue; lane 2, well- and moderately differentiated ESCC (G1-2); lane 3, poorly differentiated ESCC (G3-4); lane 4, tumor depth of invasion T1-2; lane 5, tumor depth of invasion T3-4; lane 6, lymph node metastasis-negative; lane 7, lymph node metastasis-positive (N1); lane 8, lymph node metastasis-positive (N2-3). $\beta$-actin was used as internal control.

were jointly re-evaluated by the investigators and a consensus was obtained.

Quantitative reverse-transcription polymerase chain reaction ( $q R T-P C R)$. Total RNA was extracted from ESCC cells using TRIzol reagent (Invitrogen, Carlsbad, CA, USA) according to the manufacturer's instructions and reverse-transcribed using the SuperScript III RT-PCR system (Invitrogen) according to the manufacturer's protocol. cDNA $(1 \mu \mathrm{l})$ was used for PCR amplification. The cDNA templates included the GLP fragment (179 bp) with primers 5'-TTC AAG CAA TTT TCC TGT CT-3' (forward) and 3'-CAT TAA TCC CAG CAC TTT GG-5' (reverse) and the internal control $\beta$-actin gene (314 bp) with primers 5'-TCC TGT GGC ATC CAC GAA ACT-3' (forward) and 3'-GAA GCA GCA TTT GCG GTG GAC GAT-5' (reverse). The thermal cycle reactions were performed at annealing temperatures of $57.5^{\circ} \mathrm{C}$ for EHMT1 and $55.5^{\circ} \mathrm{C}$ for $\beta$-actin, for a total of 26 cycles. The products were electrophoresed on $1.2 \%$ agarose gel containing ethidium bromide and visualized and photographed under UV light.

Statistical analysis. Data were analyzed with the SPSS software, version 17.0 (SPSS Inc., Chicago, IL, USA) and are representative of at least three independent experiments with similar results. The correlation between the EHMT1 expression and the clinicopathological parameters was assessed by the $\chi^{2}$ test and bivariate analysis. Survival curves were calculated by the Kaplan-Meier product-limit estimate method and examined with the log-rank test. The significance of multiple predictors of survival was assessed by the Cox regression analysis. $\mathrm{P}<0.05$ was considered to indicate a statistically significant difference.

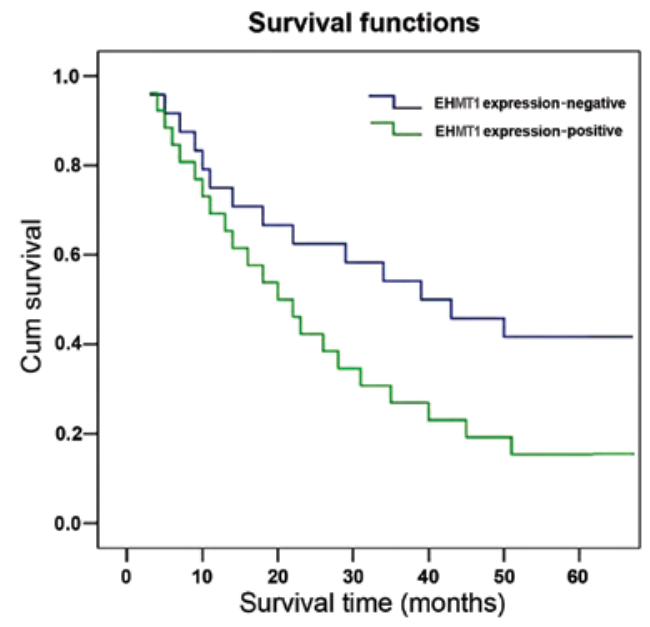

Figure 2. Kaplan-Meier curves of overall survival (OS) in esophageal squamous cell cancer (ESCC) patients. The 5-year OS rates were 41.7 and $15.4 \%$ in ESCC patients with negative $(n=24)$ and positive EHMT1 expression $(n=26)$, respectively. The difference between the EHMT1-negative and -positive expression groups was statistically significant $(\mathrm{P}=0.048)$. Cum, cumulative.

\section{Results}

EHMT1 expression in esophageal squamous preinvasive lesions and ESCC. The immunohistochemical staining for EHMT1 was most prominent in the nuclei of the ESCC cells. EHMT1 was also strongly expressed in the proliferative esophageal epithelium. However, the expression of EHMT1 was low in the normal esophageal epithelial cells (Fig. 1A). The expression of EHMT1 differed significantly between ESCC and the normal esophageal squamous epithelium (Table II).

The RT-PCR analysis indicated that the expression of EHMT1 was significantly stronger in cancer tissues compared 
to the corresponding normal tissues. At the RNA level, the overexpression of EHMT1 in human ESCC tissues was correlated with tumor grade, depth of invasion and lymph node metastasis (Fig. 1B). These findings were in concordance with the immunohistochemical data.

Association between EHMT1 expression and clinicopathological findings of ESCC. The correlation between the expression of EHMT1 and the clinicopathological characteristics of ESCC patients is summarized in Table I. The results demonstrated that the expression of the EHMT1 protein was not correlated with age and gender. However, EHMT1 expression was significantly correlated with tumor grade, depth of invasion, lymph node metastasis and tumor stage $(\mathrm{P}<0.05)$.

Survival analysis and prognostic significance of EHMT1 expression. The correlation between survival and EHMT1 expression was evaluated in the 50 cases with operable ESCC. A significant difference was observed when the patient cohort was stratified by the level of EHMT1 expressions. Of note, patients with ESCC who exhibited high expression levels of EHMT1 had a lower survival rate compared to that of patients without high EHMT1 expression $\left(\chi^{2}=3.922 ; \mathrm{P}=0.048\right)$ (Fig. 2). The multiple predictors of survival assessed by the Cox regression analysis identified EHMT1 expression as an independent prognostic factor for overall survival in ESCC patients (hazard ratio $=1.605 ; 95 \%$ confidence interval: $1.037-2.485 ; \mathrm{P}=0.034$ ) (unpublished data).

\section{Discussion}

EHMT1 is a subunit of histone methyltransferases. Although previous studies identified EHMT1 as a regulator of tumor progression (9), the clinicopathological significance of EHMT1 expression in ESCC has not been fully elucidated. In the present study, EHMT1 expression was shown to be higher in ESCC and the proliferative esophageal epithelium compared to that in normal esophageal tissues and its expression was correlated with poor prognosis. To the best of our knowledge, this is the first study on the association between EHMT1 and ESCC.

Histone H3K9 methyltransferases (EHMT1 and EHMT2), originally identified by their ability to interact with histones in cells through the methylation of heterochromatin, are considered to be crucial in maintaining the balance state of cells. EHMT1/EHMT2 may catalyze the dimethylation of $\mathrm{H} 3 \mathrm{~K} 9$, a process that represses gene transcription in euchromatic cells $(10,11)$. A previous study by Bird indicated that the methylation of H3K9 may exert an effect on DNA methylation, directly or indirectly (12). H3K9 methylation directs $\mathrm{CpNpG}$ methylation and was shown to be necessary for DNA methylation in fungi $(13,14)$. DNA methylation is crucial in the regulation of gene expression and chromatin organization within normal eukaryotic cells. EHMT1/EHMT2 is also required for the maintenance of DNA methylation at endogenous retrotransposons, imprinted loci and other genes present in differentiated cells (15). A recent study also revealed that EHMT1 is required for the DNA methylation of cancer germline and genomic DNA in mouse embryonic stem cells (16). The direct effect reverse to methylation is demethylation; however, histone methylation is considered to be irreversible and, once it occurs, such a change may be present for the entire cell life span and may even be transferred to the next generation. In cancer cells, alterations of the global patterns of DNA methylation are considered to be normal (17). DNA hypermethylation of certain CIMP (colorectal tumors with a $\mathrm{CpG}$ island methylator phenotype)-associated gene promoters was detected during the early stages of colorectal tumorigenesis (18). Moreover, promoter DNA methylation of the ERG gene is a common event in human prostate cancer (19). It was also proven that aberrant DNA methylation is associated with chronic myelogenous leukemia progression and that DNA methylation may be a marker associated with imatinib resistance (20). These results suggested an association between histone methylation and cancer progression.

In our study, we selected 50 cases of patients with operable ESCC. The results confirmed that the expression of EHMT1 was higher in ESCC and the atypical hyperplastic esophageal epithelium compared to that in normal esophageal tissues, by using immunohistochemistry and RT-PCR. EHMT1 may be a key molecular marker for the progression of human ESCC. Moreover, EHMT1 expression was also associated with tumor grade, depth of invasion, lymph node metastasis and tumor stage.

It was also verified that the EHMT2 expression pattern in ESCC was similar to the EHMT1 expression pattern, using immunohistochemistry with an anti-EHMT2 antibody (1:80 dilution; BD Biosciences, Franklin Lakes, NJ, USA (unpublished data). This confirmed that in ESCC or atypical hyperplastic esophageal squamous epithelia, the expression level of each histone methyltransferase subunit may rise aberrantly and the process of histone methylation becomes disordered.

Our study also demonstrated that the expression of EHMT1 was positively correlated with lower survival in ESCC. Therefore, the expression of EHMT1 may serve as a prognostic factor for predicting the outcome of ESCC patients. Thus, the detection of EHMT1 aberrations may be a useful biomarker to identify ESCC patients with poor prognosis.

In conclusion, our data indicated that EHMT1 expression is elevated in human ESCC and may play a significant role in human ESCC progression. Our analysis of clinical studies also demonstrated that the expression of EHMT1 may be a useful biomarker predicting ESCC progression and prognosis.

\section{Acknowledgements}

This study was supported by the National Nature Science Foundation of China (no. 81272605).

\section{References}

1. Thallinger CM, Kiesewetter B, Raderer M and Hejna M: Preand postoperative treatment modalities for esophageal squamous cell carcinoma. Anticancer Res 11: 4609-4627, 2012.

2. Tachibana M, Ueda J, Fukuda M, Takeda N, Ohta T, Iwanari H, Sakihama T, Kodama T, Hamakubo T and Shinkai Y: Histone methyltransferases G9a and GLP form heteromeric complexes and are both crucial for methylation of euchromatin at $\mathrm{H} 3-\mathrm{K} 9$. Genes Dev 19: 815-826, 2005.

3. Collins R and Cheng X: A case study in cross-talk: the histone lysine methyltransferases G9a and GLP. Nucleic Acids Res 38: 3503-3511, 2010. 
4. Chin HG, Esteve PO, Pradhan M, Benner J, Patnaik D, Carey MF and Pradhan S: Automethylation of G9a and its implication in wider substrate specificity and HP1 binding. Nucleic Acids Res 35: 7313-7323, 2009.

5. Rathert P, Dhayalan A, Murakami M, Zhang X, Tamas R, Jurkowska R, Komatsu Y, Shinkai Y, Cheng X and Jeltsch A: Protein lysine methyltransferase G9a acts on non-histone targets. Nat Chem Biol 4: 344-346, 2008.

6. Pless O, Kowenz-Leutz E, Knoblich M, Lausen J, Beyermann M, Walsh MJ and Leutz A: G9a-mediated lysine methylation alters the function of CCAAT/enhancer-binding protein-beta. J Biol Chem 283: 26357-26363, 2008.

7. Fritsch L, Robin P, Mathieu JR, et al: A subset of the histone H3 lysine 9 methyltransferases Suv39h1, G9a, GLP, and SETDB1 participate in a multimeric complex. Mol Cell 37: 46-56, 2010.

8. Sampath SC, Marazzi I, Yap KL, Sampath SC, Krutchinsky AN, Mecklenbrauker I, Viale A, Rudensky E, Zhou MM, Chait BT and Tarakhovsky A: Methylation of a histone mimic within the histone methyltransferase G9a regulates protein complex assembly. Mol Cell 27: 596-608, 2007.

9. Lee SH, Kim J, Kim WH and Lee YM: Hypoxic silencing of tumor suppressor RUNX3 by histone modification in gastric cancer cells. Oncogene 28: 184-194, 2009.

10. Purcell DJ, Khalid O, Ou CY, et al: Recruitment of coregulator G9a by Runx 2 for selective enhancement or suppression of transcription. J Cell Biochem 113: 2406-2414, 2012.

11. Purcell DJ, Jeong KW, Bittencourt D, et al: A distinct mechanism for co-activator versus corepressor function by histone methytransferase G9a in transcriptional regulation. J Biol Chem 286: 41963-41971, 2011.

12. Bird A: Methylation talk between histones and DNA. Science 294: 2113-2115, 2001
13. Tamaru H, Zhang X, McMillen D, et al: Trimethylated lysine 9 of histone $\mathrm{H} 3$ is a mark for DNA methylation in Neurospora crassa. Nat Genet 34: 75-79, 2003.

14. Jackson JP, Lindroth AM, Cao X and Jacobsen SE: Control of CpNpG DNA methylation by the KRYPTONITE histone H3 methyltransferase. Nature 416: 556-560, 2002.

15. Ikegami K, Iwatani M, Suzuki M, et al: Genome-wide and locus-specific DNA hypomethylation in G9a deficient mouse embryonic stem cells. Gene Cells 12: 1-11, 2007.

16. Chen L, Li Z, Zwolinska AK, et al: MDM2 recruitment of lysine methyltransferases regulates p53 transcriptional output. EMBO J 29: 2538-2552, 2010.

17. Candelaria M, de la Cruz-Hernandez E, Taja-Chayeb L, et al: DNA methylation-independent reversion of gemcitabine resistance by hydralazine in cervical cancer cells. PloS One 7: e29181, 2010

18. Ibrahim AE, Arends MJ, Silva AL, Wyllie AH, Greger L, Ito Y, Vowler SL, Huang TH, Tavare S and Murrell A: Sequential DNA methylation changes are associated with DNMT2B overexpression in colorectal neoplastic progression. Gut 60: 499-508, 2010.

19. Schwartzman J, Mongoue-Tchokote S, Gibbs A, et al: A DNA methylation microarray-based study identifies ERG as a gene commonly methylated in prostate cancer. Epigenetics 6: 1248-1256, 2011.

20. Jelinek J, Gharibyan V, Estecio MR, et al: Aberrant DNA methylation is associated with disease progression, resistance to imatinib and shortened survival in chronic myelogenous leukemia. PloS One 6: e22110, 2011. 